\title{
Decoding DNA labels by melting curve analysis using real-time PCR
}

\author{
József A. Balog ${ }^{1,2}$, Liliána Z. Fehér ${ }^{3}$, and László G. Puskás ${ }^{1,2,3}$ \\ ${ }^{1}$ Avicor Ltd., Szeged, Hungary, ${ }^{2}$ Biological Research Center, Hugarian Academy of Sciences, Institute of \\ Genetics, Szeged, Hungary, and ${ }^{3}$ AVIDIN Ltd., Szeged, Hungary
}

BioTechniques 63:261-266 (December 2017) doi 10.2144/000114618

Keywords: DNA coding; real-time PCR; melting curve analysis

Supplementary material for this article is available at www.BioTechniques.com/article/114618.

Synthetic DNA has been used as an authentication code for a diverse number of applications. However, existing decoding approaches are based on either DNA sequencing or the determination of DNA length variations. Here, we present a simple alternative protocol for labeling different objects using a small number of short DNA sequences that differ in their melting points. Code amplification and decoding can be done in two steps using quantitative PCR (qPCR). To obtain a DNA barcode with high complexity, we defined 8 template groups, each having 4 different DNA templates, yielding $15^{8}$ (>2.5 billion) combinations of different individual melting temperature $\left(T_{m}\right)$ values and corresponding ID codes. The reproducibility and specificity of the decoding was confirmed by using the most complex template mixture, which had 32 different products in 8 groups with different $T_{m}$ values. The industrial applicability of our protocol was also demonstrated by labeling a drone with an oil-based paint containing a predefined DNA code, which was then successfully decoded. The method presented here consists of a simple code system based on a small number of synthetic DNA sequences and a cost-effective, rapid decoding protocol using a few qPCR reactions, enabling a wide range of authentication applications.

Today, it is increasingly difficult for a customer to define the origin of a product. Adulteration is a worldwide problem that is expanding to nearly every sort of product, from the cheapest to the most expensive. For this reason, identification marking is especially important for unique industrial or military products. There are many different methods for labeling a product, such as barcoding and fluorescent labeling, but most of these labels are not unique enough to protect the product against falsification. Moreover, labels must be invisible, cheap, tolerant of environmental conditions, easily detectable, and non-reactive with the product material. It is difficult to meet all of these requirements; however the use of nucleic acids, especially DNA, could provide a solution.
Unique DNA sequences from living organisms are frequently used for taxonomic identification. DNA sequencing is a suitable method for determining the composition of foodstuffs of animal and plant origin. Wallace et al. used the internal transcribed spacers of nuclear ribosomal genes to distinguish Korean and American ginseng species (1). In another study, a fragment of the mitochondrial cytochrome $\mathrm{C}$ oxidase 1 gene was proven to be an appropriate barcode for identification of the origin of fish and fish-derived foodstuffs $(2,3)$. In addition to natural DNA, synthetic DNA labels have been used for diverse applications. Synthetic DNA can be a valuable tracer in groundwater investigations if unique DNA fragments are injected into an aquifer. The tracers can be detected by PCR and DNA sequence analysis (4). Furthermore, Cox (5) described a method for barcoding objects with DNA. In that study, many DNA templates of different lengths were mixed together and used to label a letter. To decode the label barcode, the DNA mixture was eluted from the letter, amplified by PCR, and the amplified templates were resolved by agarose gel electrophoresis. Synthetic DNA labels may also be useful for protecting food products. Puddu et al. described a technique that is suitable for tagging olive oil. DNA fragments were adsorbed onto the surfaces of magnetic nanoparticles $\left(\mathrm{Fe}_{2} \mathrm{O}_{3}\right)$, and this complex was coated with a dense silica layer. The decoding was based

\section{METHOD SUMMARY}

For high complexity DNA barcoding, eight template groups, each having four DNA templates with different melting points, were defined. Synthetic templates and primers were designed for minimal homology with bacterial, plant, and animal sequences, minimizing environmental background interference. Code amplification and decoding were performed in subsequent steps by melting curve analysis using real-time PCR. 
on magnetic separation, and the DNA fragments were analyzed by quantitative PCR (qPCR) and Sanger sequencing (6). Another successful application of this silica particles with encapsulated DNA (SPED) technique was presented by Bloch et al. (7). The authors labeled milk with SPED and subsequently manufactured yogurt and cheese from the milk. The extraction of the marker from the milk-derived foodstuff was performed by washing/centrifugation. The specific DNA code was quantified by qPCR.

Here, we present an alternative method for labeling objects with artificial DNA. Our code system is based on synthetic DNA fragments with different melting temperature $\left(T_{m}\right)$ values. Code amplification and decoding can be performed in subsequent steps using qPCR. To demonstrate the applicability of our protocol, we labeled different surfaces with a DNA mixture having a defined combination of $T_{m}$ values. Labeling, specific amplification, and decoding were successfully performed on a drone's surface.

\section{Materials and methods \\ Primers and templates}

To create our coding system, eight primer pairs were designed to form eight separate groups of synthetic DNA. All sequences were synthesized at Bioneer (Bioneer Corp., Daejon, South Korea). The templates and primers were designed to show minimal homology with bacterial, plant, and animal sequences when assessed by BLAST (https://blast.ncbi.nlm.nih.gov/ Blast.cgi). Each primer pair was used with four specific templates (A, B, C, D), each having a different $C G$ content and length. The sequences of the primers and the single-stranded DNA templates used in the study are listed in Table 1.

$T_{m}$ measurement by qPCR

and melting curve analysis

The amplification and melting curve analysis were performed in $20 \mu \mathrm{l}$ reactions in a Light Cycler Nano Instrument (Roche Diagnostics Corp., Indianapolis, IN). All reactions consisted of $250 \mathrm{nM}$ of each primer, $10 \mu \mathrm{l}$ FastStart Essential DNA Green Master 2× (Roche Diagnostics), $8 \mu \mathrm{l}$ PCR-grade water, and $0.1 \mathrm{pM}$ of each template. A negative control (water without DNA) reaction

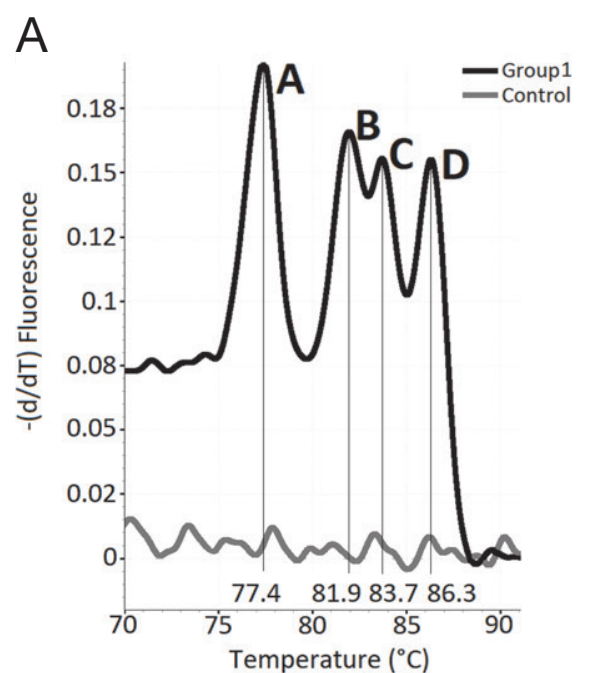

\begin{tabular}{|c|c|c|}
\hline$\#$ & Tm code & ID code \\
\hline 1 & A & 1 \\
\hline 2 & B & 2 \\
\hline 3 & C & 3 \\
\hline 4 & D & 4 \\
\hline 5 & $A B$ & 5 \\
\hline 6 & $A C$ & 6 \\
\hline 7 & $A D$ & 7 \\
\hline 8 & $B C$ & 8 \\
\hline 9 & BD & 9 \\
\hline 10 & CD & 0 \\
\hline 11 & $A B C$ & $a$ \\
\hline 12 & ABD & b \\
\hline 13 & ACD & c \\
\hline 14 & BCD & d \\
\hline 15 & ABCD & e \\
\hline
\end{tabular}

C Templates

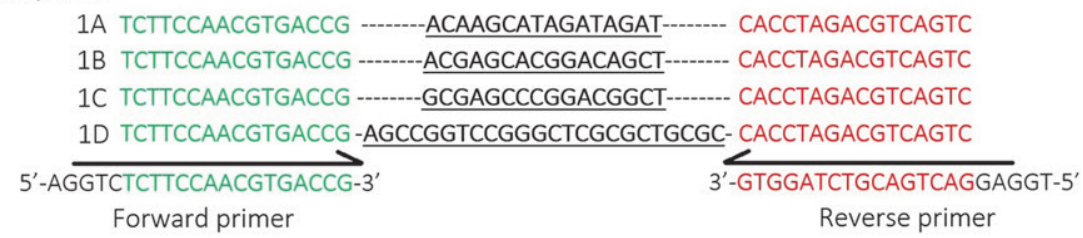

Figure 1. Primer and template sequences of one template group and their corresponding melting temperature $\left(T_{m}\right)$ values and ID codes. (A) The negative first derivative of the fluorescence vs. temperature $(-d F / d T)$ dissociation curve of the first template group having four different $T_{m}$ values as assessed by melting curve analysis after qPCR amplification. (B) The $T_{m}$ code and corresponding ID code are defined by sequence variations in one template group consisting of four different templates with different $T_{m}$ values. (C) The forward primer binding site of the template is highlighted in green, while the reverse primer binding site is highlighted in red. The black bold and underlined sequences differ in length (A,B,C: 48 nucleotides; D: 56 nucleotides) and CG content, ensuring differences in $T_{m}$ (CG content: $1 A-47.9 \%$; 1B-58.3\%; 1C-64.6\%; 1D-67.9\%).

was included in all experiments. The cycling parameters were as follows: enzyme activation at $95^{\circ} \mathrm{C}$ for $10 \mathrm{~min}$, denaturation at $95^{\circ} \mathrm{C}$ for $15 \mathrm{~s}$, annealing at $58^{\circ} \mathrm{C}$ for $25 \mathrm{~s}$, and extension at $72^{\circ} \mathrm{C}$ for 25 s. To avoid over-amplification of the products, the cycling was terminated just after the reactions reached plateau (i.e., at 10-14 cycles). The amplification was followed by the melting curve measurement: rapid heating of amplified samples to $95^{\circ} \mathrm{C}$ to denature the DNA; cooling to $60^{\circ} \mathrm{C}$ to let DNA strands to anneal; then slowly heating the samples to $95^{\circ} \mathrm{C}$, where the change in fluorescence was measured at each $0.1^{\circ} \mathrm{C}$ rise for $1 \mathrm{~s}$.

\section{Paint preparation}

Oil paint (Permanent Blue; PannonColor Ltd., Budapest, Hungary) (400 mg) was added to a mixture of 300 $\mu$ l acetone, $200 \mu$ turpentine, and 10 $\mu \mathrm{l}$ 25\% (w/v) DNA Stabilization Resin Solution (Cat. \#DSRS-01; Avicor Ltd, Szeged, Hungary). The mixture was vortexed for $1 \mathrm{~min}$, and the predefined DNA code (1B-2B-3ABCD) was added to the mixture. The concentrated DNA code mixture contained $100 \mathrm{pM}$ of each template. The surface of the drone was labeled with 10 drops (10 $\mu$ l each) of the mixture.

\section{DNA extraction from paint}

Seventy-two hours after marking, the dried paint drops were separated from the surface of a drone (Bonn Hungary Electronics, Budapest, Hungary) with a clear razor blade. For the long-term stability test, paint drops from a plastic surface (Cell culture dish, Cat. \#430167; Corning Inc., Corning, NY) were recovered after 6 months of storage at room temperature by a similar method. Oil paint $(30 \mathrm{mg}$ ) was dissolved in a mixture of $200 \mu \mathrm{l}$ PCR-grade water and $200 \mu \mathrm{l}$ ethyl acetate. The solution was vortexed for $1 \mathrm{~min}$ and then centrifuged for 2 min at 10,000 $\times g$. The bottom layer was pipetted to a new tube with $200 \mu \mathrm{l}$ ethyl acetate and centrifuged for 2 min 
Table 1: Primer and template sequences.

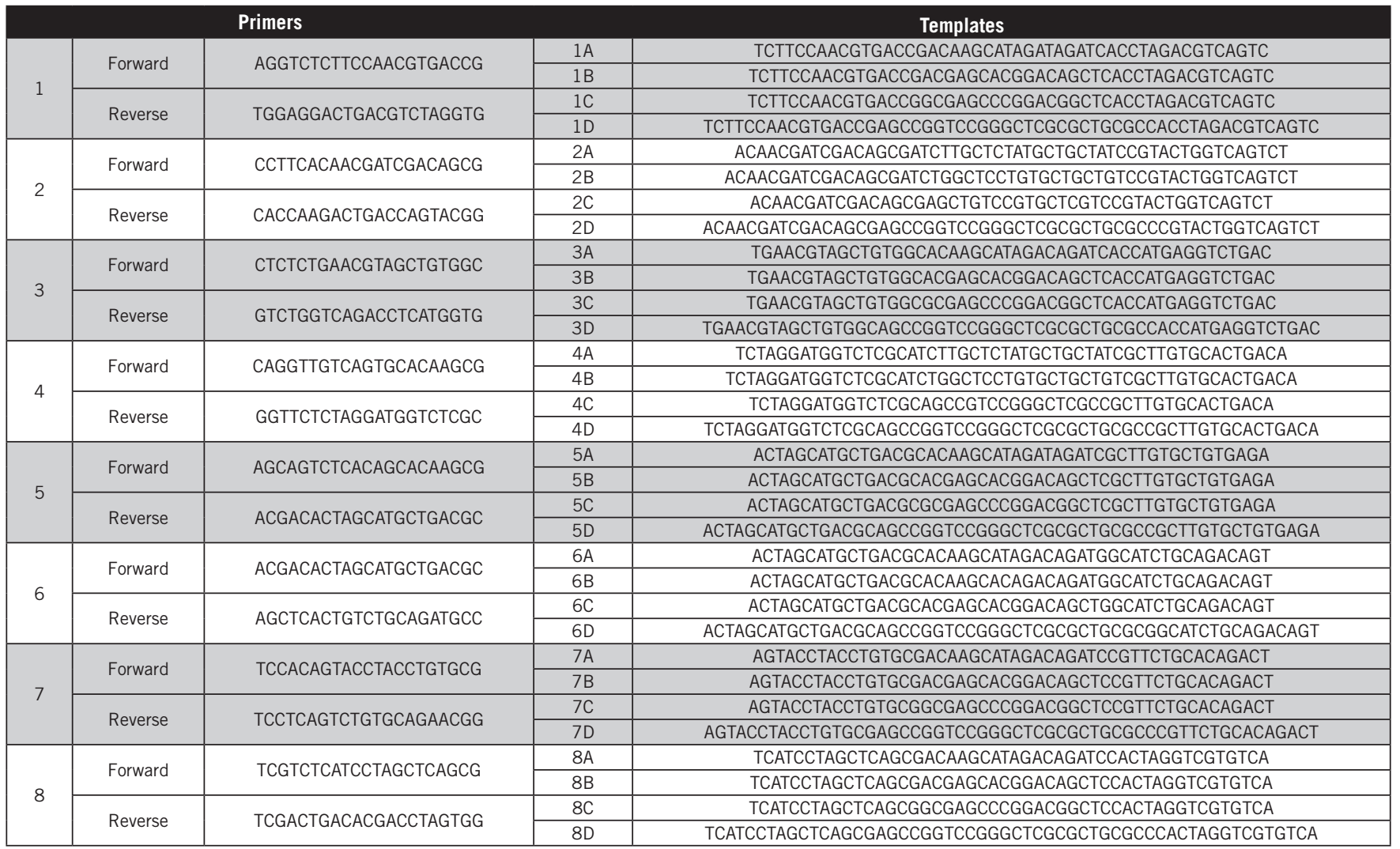

at $10,000 \times g$. Five microliters from the lower, aqueous phase containing the DNA templates was used for decoding by qPCR. To detect the presence of each template, an individual qPCR was run with each primer combination.

\section{Results and discussion}

Most DNA-based coding technologies have been based on sequence or length variations of the DNA. Here, we present a novel approach based on variations in $T_{m}$ values of individual templates, which could be measured by melting curve analysis using any qPCR instrument. Since the precise $T_{m}$ of a DNA template is influenced by several factors (salt concentration, PCR reagents, melting profile, etc.), we defined groups of DNA templates where each group has only a maximum of four different sequences defining four different $T_{m}$ values (Figure 1A). If we calculate the possible combinations of the $4 T_{m}$ values (or $T_{m}$ codes), 15 different combinations can be achieved if we exclude the one having no signal (containing no DNA template) in the group (Figure 1B). To refer to each $T_{m}$ code in a template group, we defined an ID code
(Figure 1B) that was used in subsequent DNA coding as a DNA barcode digit. The sequences of the four templates in one group are designed such that both the 5'and 3 '- flanking regions are the same in order to enable the amplification of all of the templates in the group by one primer pair (Figure 1C and Table 1).

To obtain a high complexity among the DNA barcodes, we defined 8 template

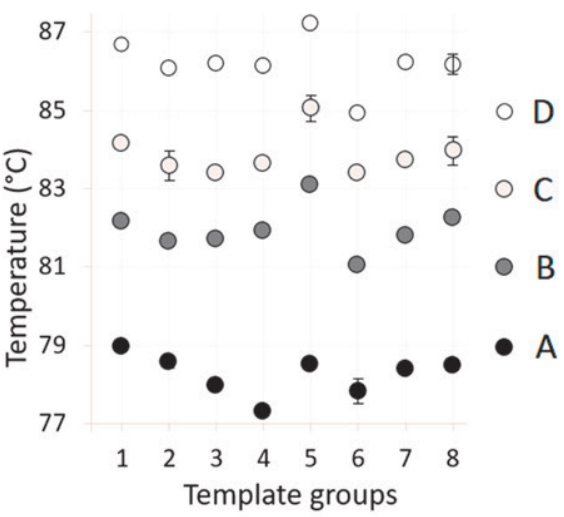

Figure 2. Melting temperature $\left(T_{m}\right)$ measurements for the $\mathbf{3 2}$ templates. The $T_{m}$ was determined for each of the DNA templates for all of the eight template groups. Individual reactions contained only one template sequence. Mean and SD values were determined from nine independently repeated qPCR reactions. Only SD values $>0.25$ are depicted in the graph. groups, each having 4 different DNA sequences, which enabled us to define $15^{8}$ possible combinations, meaning 2,562,890,625 different individual $T_{m}$ (and corresponding ID) codes. To confirm the reproducibility of the $T_{m}$ values for each DNA template, $8 \times 4=32$ individual qPCRs were run, with 9 repetitions. The average $T_{m}$ values with their standard deviations (SD) are shown in Figure 2. As expected, very high reproducibility could be achieved. The melting points of the designed templates were well separated and could be clearly detected within a 0.5 degree deviation. $T_{m}$ values from individual reactions were used as the reference $T_{m}$ values in experiments where DNA template mixtures were used.

To demonstrate the utility of our approach, we prepared different template mixtures containing different DNA sequences that determined each $T_{m}$ code for all eight of the template groups. At first, the simplest combination code mixture was prepared, containing only 1 template in each of the 8 template groups. That $T_{m}$ code was the following: $D, A, C, B, A, B, C, D$. This can be translated into the following ID code using the table shown in Figure 1B: 41321234. All of the 

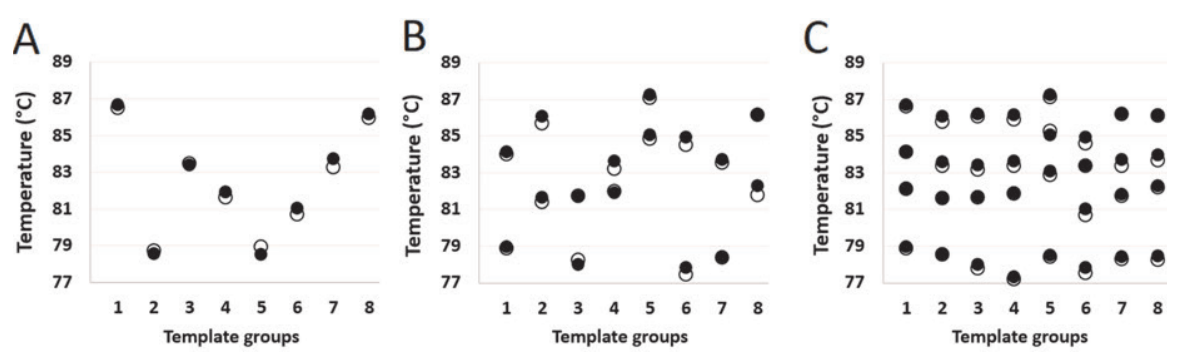

$T_{m}$ values - and therefore the $T_{m}$ codes and the ID codes - could be determined without any error. When the $T_{m}$ values obtained from individual reactions were plotted with those obtained from the eight template mixtures, no significant difference could be detected (Figure 3A).

Next, we prepared an ID code (69580769) that is defined by two templates in each template group $\left(T_{m}\right.$ code: AC-BD-AB-BC-CD-AD-AC-BD). Similar to the previous experiment, the $T_{m}$ points, and therefore the DNA barcodes, could be determined with high precision (Figure 3B).

Finally, the most complex mixture was prepared: each of the eight template groups contained all four of the different DNA templates, which corresponds to "ABCD" in the $T_{m}$ code and to "e" in the ID code. Even from this mixture, which contained 32 different templates together, all of the $T_{m}$ values could be determined correctly, with small deviations from the reference $T_{m}$ values (Figure $3 C$ ).

These results clearly demonstrate that the $T_{m}$ for any template combination can be determined; thus, any ID code can be decoded. A schematic of our protocol is shown in Figure 4. One advantage of the protocol is that the $T_{m}$ measurement is done with the same qPCR instrument used for the amplification. Because of the high amplification capability of PCR, even a minute amount of DNA code can be decoded.

To demonstrate the utility of our protocol for industrial applications, we prepared an oil-based paint containing DNA templates and used it to label a drone. First, a three-digit ID code (22e) was generated, and DNA templates according to the $T_{m}$ code $(B, B, A B C D)$ were mixed equally and then spiked into the paint. The coded paint, was then pipetted onto the body of the drone in 10- $\mu$ l drops. For decoding the DNA barcode 3 days later, the paint dots were scraped off, dissolved, and extracted. The aqueous phase containing the DNA templates was amplified by qPCR in three separate reactions, each having primer pairs specific to its template group. After obtaining the melting curves, the $T_{m}$
Figure 3. T code determination from a mixture containing DNA from all eight DNA template groups. The $T_{m}$ values were determined for each DNA template group (1 to 8 ) in a separate qPCR using a template mixture containing one $(A)$, two $(B)$, or four (C) DNA templates in all eight groups. Black dots represent $T_{m}$ values determined from individual reactions containing only one DNA sequence (see details in Figure 1). Empty dots represent $T_{\text {}}$ values determined from a template containing all of the template sequences from the eight groups.

codes could be recovered. Decoding of the DNA barcode was successful (data not shown).

To ensure compatibility of the DNA barcodes with oil-based paint, we recovered DNA from non-solidified oil paint 1 day and 10 days after application. No significant alteration in $\mathrm{C}_{\mathrm{q}}$ values for the 2 samples could be detected after qPCR (11.67 versus 10.61 and 9.97 versus 10.81 for samples D1 and D10, respectively). To confirm the long-term stability of the synthetic DNA mixture in paint, different surfaces (metal, wood, paper, and plastic) were labeled and the labels were successfully decoded by melting curve analyses, even after 6 months. DNA recovery and GPCR decoding from old paint spots on plastic surfaces using five codes in quadruplicate were performed. Only moderate degradation could be detected, but interestingly, a sequence-dependent degradation profile was observed (Supplementary Figure S1). In two cases no significant alteration in $\mathrm{C}_{\mathrm{q}}$ values was found (codes \#1 and \#2). For the other

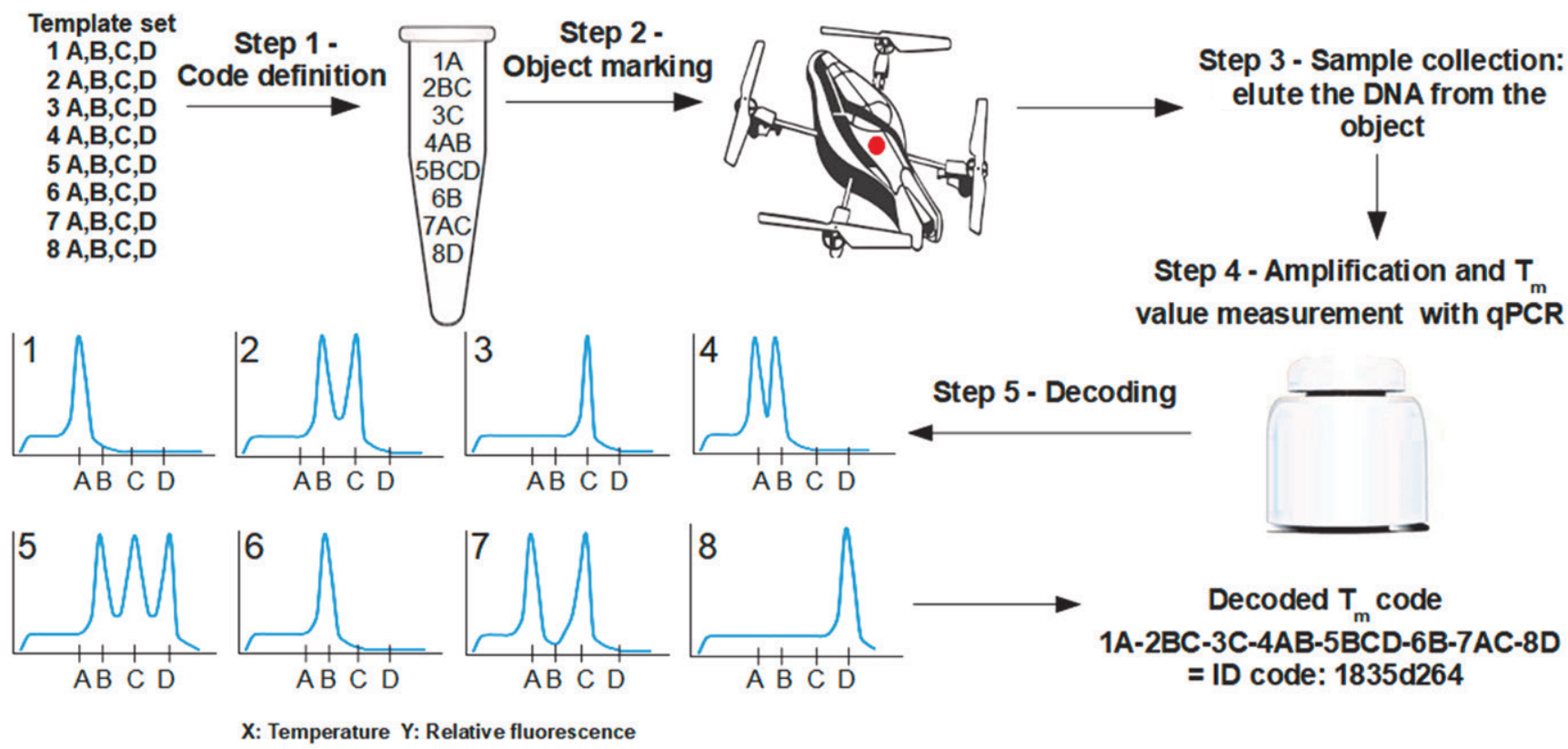

Figure 4. Schematic of the DNA coding approach and the decoding protocol with melting curve analysis using a qPCR instrument. 
